\title{
Stereotactic body radiotherapy for central lung tumours
}

\author{
M DAHELE, MBChB, PhD, H TEKATLI, MD and S SENAN, MBBS, PhD \\ Department of Radiation Oncology, VU University Medical Center, Amsterdam, Netherlands \\ Address correspondence to: Dr Max Dahele \\ E-mail: m.dahele@vumc.nl
}

(The Editors do not hold themselves responsible for opinions expressed by correspondents)

To the Editor,

We read with interest the recent article by Adebahr et al, ${ }^{1}$ "LungTech, an EORTC Phase II trial of stereotactic body radiotherapy for centrally located lung tumours: a clinical perspective". There are now several series reporting acceptable toxicity and good outcomes for moderately central lung tumours treated with stereotactic body radiotherapy (SBRT) ${ }^{2-5}$ And in a recent survey, 23 of 30 selected European centres indicated that they were treating tumours within $2 \mathrm{~cm}$ of the central mediastinal structures. ${ }^{6}$ In addition, more detailed descriptions of central tumour location and planned radiation doses to the treatment volume and organs at risk are beginning to emerge. ${ }^{4,5}$ One of the aims of the LungTech study (https://clinicaltrials.gov/ct2/ show/NCT01795521) was to determine the toxicity of central lung SBRT delivered in eight fractions of $7.5 \mathrm{~Gy}$. However, as the authors acknowledge, not all central tumours can be considered equal, and although the definitions remain imprecise, a distinction is generally made between moderately central and very central locations. ${ }^{5}$ These have been considered to represent lower and higher risk scenarios, respectively, although the absolute risks, in particular of very central lung SBRT, are incompletely quantified and expected to vary with the dose and fractionation of the treatment. ${ }^{2}$ LungTech explicitly excludes very central tumours and limits the dose that the central airways can receive to about $75 \%$ of the prescription dose. Its focus may therefore reasonably be considered to be on moderately central lung SBRT.

The article suggests that the study prescription dose and the dose constraint for the central airways are based on the VU University Medical Center's experience. ${ }^{3}$ The airway constraint is an important parameter in defining central lung SBRT treatment philosophy-if the airway dose is tightly constrained, then very central tumours cannot be treated.
While we cannot go into detail in a letter, we appreciate this opportunity to briefly clarify both the attributed central airway constraint and our general approach to central lung SBRT. Although the figure that they have quoted for the central airway constraint (eight fractions of $5.5 \mathrm{~Gy}$ ) is correct, in our centre (VU University Medical Center), this is a maximum point dose limit (not $0.5 \mathrm{~cm}^{3}$ ), and it is only used as a guide for treatment planning when there is no overlap between the planning target volume (PTV) and central airways. When there is an overlap between the PTV and selected central structures such as the airways, then as previously stated "Dose reductions of the PTV to spare overlapping critical structures were not used". 3 However, although such patients are not excluded from receiving eight-fraction lung SBRT, in practice, we are more conservative about exposing the most central portion of the airways - the proximal part of the main bronchus, the carina complex and the trachea-to the full dose. This is one reason why so far the majority of patients who we and others have treated with central lung SBRT have moderately central target volumes, and the number of patients in whom the most central portion of the airways has been exposed to the full 60 Gy (or more) in eight fractions is small. ${ }^{3,5}$

Finally, although LungTech has not been specifically designed to find the limits of central lung SBRT, the RTOG 0813 study (https://clinicaltrials.gov/show/NCT00750269) will hopefully contribute to our understanding of this, although this will ultimately depend on the number of patients enrolled and treated who have very central tumours.

\section{CONFLICTS OF INTEREST}

The VU University Medical Center Department of Radiation Oncology has a research agreement with Varian Medical Systems. MD and SS have received honoraria and travel support from Varian Medical Systems. MD has received honoraria and travel support from Brainlab. 


\section{REFERENCES}

1. Adebahr S, Collette S, Shash E, Lambrecht M, Le Pechoux C, Faivre-Finn C, et al. LungTech, an EORTC phase II trial of stereotactic body radiotherapy for centrally located lung tumours: a clinical perspective. $\mathrm{Br} J$ Radiol 2015; 88: 20150036. doi: 10.1259/ bjr.20150036

2. Senthi S, Haasbeek CJ, Slotman BJ, Senan S. Outcomes of stereotactic ablative radiotherapy for central lung tumours: a systematic review. Radiother Oncol 2013; 106: 276-82. doi: 10.1016/j.radonc.2013.01.004
3. Haasbeek CJ, Lagerwaard FJ, Slotman BJ, Senan S. Outcomes of stereotactic ablative radiotherapy for centrally located early-stage lung cancer. J Thorac Oncol 2011; 6: 2036-43. doi: 10.1097/JTO.0b013e31822e71d8

4. Chang JY, Li QQ, Xu QY, Allen PK, Rebueno N, Gomez DR, et al. Stereotactic ablative radiation therapy for centrally located early stage or isolated parenchymal recurrences of non-small cell lung cancer: how to fly in a "no fly zone". Int J Radiat Oncol Biol Phys 2014; 88: 1120-8. doi: 10.1016/j.ijrobp.2014.01.022
5. Chaudhuri AA, Tang C, Binkley MS, Jin M, Wynne JF, von Eyben R, et al. Stereotactic ablative radiotherapy (SABR) for treatment of central and ultra-central lung tumours. Lung Cancer 2015; 89: 50-6. doi: 10.1016/j. lungcan.2015.04.014

6. Dahele M, Hatton M, Slotman B, Guckenberger M. Stereotactic body radiotherapy: a survey of contemporary practice in six selected European countries. Acta Oncol Mar 2015: 1-5. Epub ahead of print. doi: 10.3109/ 0284186X.2014.1003961 